\title{
Numerical studies of variations in the gap and finger width ratio and travelled distance for the driving force of a radio-frequency microelectromechanical system device using the dual boundary element method
}

\author{
Y-S Liao ${ }^{1}$, S-W Chyuan ${ }^{1 *}$ and J-T Chen ${ }^{2}$ \\ ${ }^{1}$ Graduate Institute of Mechanical Engineering, National Taiwan University, Taiwan, Republic of China \\ ${ }^{2}$ Department of Harbor and River Engineering, National Taiwan Ocean University, Taiwan, Republic of China
}

\begin{abstract}
For the comb-drive design of microelectromechanical systems (MEMSs), the driving force due to the electrostatic field is very important, and an accurate electrostatic analysis is essential and indispensable. For various gaps, finger width ratios and travelled distances of the comb drive of MEMSs, the dual boundary element method (DBEM) has become a better method than the domaintype finite element method because the DBEM can provide a complete solution in terms of boundary values, with substantial saving in modelling effort. In this article, the DBEM is used to simulate the fringing field around the edges of the fixed and movable fingers of the comb drive of an MEMS for diverse design cases, and many electrostatic problems for typical comb drive designs of MEMSs are analysed, investigated and compared with a widely used approximate method. Results show that the driving force is obviously dependent on the travelled distance, and the approximate method cannot work well for all travelled positions because there is an apparent error (not less than 10 per cent), especially at the beginning and ends of the range of travel. In addition, the smaller the gap between movable and fixed fingers, the larger the driving force is, and the error of approximate method also becomes more and more predominant as the gap decreases. The results also demonstrate that the difference between the DBEM and the approximate method effect due to finger width ratio is very small. Using the DBEM presented in this article, an accurate and reasonable electrostatic field can be obtained, and the follow-up control method of driving force for the comb drive of an MEMS can be implemented more precisely.
\end{abstract}

Keywords: microelectromechanical systems (MEMSs), electrostatic, dual boundary element method (DBEM), comb-drive, driving force, gap, finger width ratio

\section{INTRODUCTION}

Until now, many new fabrication techniques have developed which helped to attain widespread use of microelectromechanical systems (MEMSs), and various devices have been reported in industry. One such area is microwave and millimetre-wave systems. MEMS technology for microwave applications should resolve many problems of high-frequency technology for wireless communications. The noticeable development of perso-

\footnotetext{
The MS was received on 12 January 2004 and was accepted after revision for publication on 30 June 2004.

* Corresponding author: Graduate Institute of Mechanical Engineering, National Taiwan University, Taipei, Taiwan, ROC. E-mail: yeaing@, iris.seed.net.tw
}

nal communication devices has forced the market to acquire miniaturized efficacious devices, which is possible only by the development of radio-frequency (RF) MEMSs [1]. Micromachining or MEMS technology is now rapidly emerging as an enabling technology to yield a new generation of high-performance RF-MEMS passives to replace off-chip passives in wireless communications [2,3]. RF-MEMSs have been demonstrated to operate from around $1 \mathrm{GHz}$ to frequencies approaching $100 \mathrm{GHz}$, and a variety of RF MEMS devices including switches, variable capacitors, resonators and filters has appeared since the late 1990s [4, 5]. Among diverse RFMEMS devices, the RF-MEMS tunable capacitors offer a solution to the shortcomings of semiconductor varactors, because the most important drawback of a semiconductor varactor is the inherent dependence of 


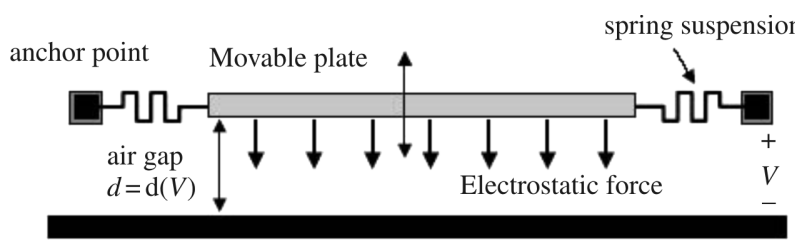

Fixed bottom plate

Fig. 1 Schematic representation of a parallel-plate tunable RF-MEMS capacitor [2]

the capacitance on the RF signal power, which makes the component behave highly non-linearly [6]. RFMEMS tunable capacitors further promise low noise and the ability to keep the signal circuit separate from the control circuit, which greatly simplifies the bias circuit [5]. Another important advantage of the RFMEMS tuneable capacitor is the integration capability with high- $Q$ inductors in a passive integration scheme, something which is generally not achievable in semiconductor technology. In essence, the capacitance of an RF-MEMS capacitor can be varied either by changing the gap spacing of the parallel plate shown in Fig. 1, or by changing the overlap area of comb-like structure (comb drive) shown in Fig. 2 [2]. Besides the RF-MEMS capacitor, using an electrostatic comb drive actuator in micromachined RF filters and other devices is preferred because the non-linear response characteristics of the parallel-plate actuator can cause frequency instability in the filter operation [1]. Because the comb drive is an import component for some RF MEMS devices, a comprehensive model to predict the physical behaviour of a comb drive, like that for RF-MEMS switches [7], is needed.

A comb drive of an MEMS as shown in Fig. 3 usually has two sets of fingers. The finger that is connected to the substrate is called the fixed finger (stationary

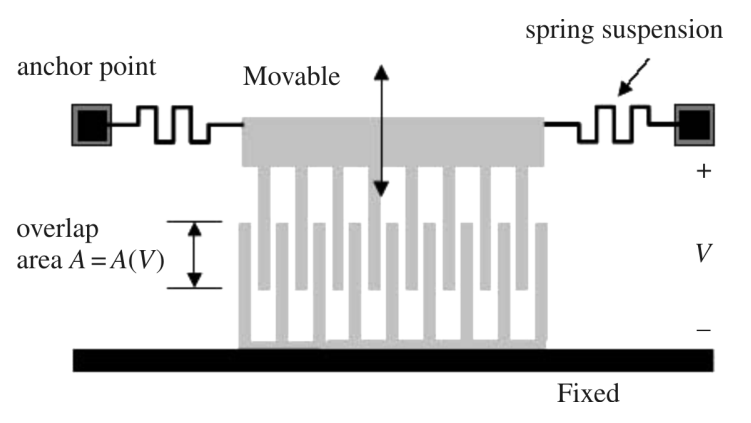

Fig. 2 Schematic representation of a comb-like structure tunable RF-MEMS capacitor [2]

electrode), and the other which is released from the substrate is called the movable finger. When two different voltages are applied to these two sets of fingers, the resulting electrostatic force drives the movable fingers towards the fixed fingers. Thus, motion is produced by this comb drive in the direction of the movement of the movable fingers [8]. Because the comb drive can be designed for either an electrostatic actuator or capacitive sensing, it has become a very important device in MEMSs [9]. Basically the in-plane interdigitated comb drives are used in in-plane or small out-ofplane or torsional motions [10], and asymmetric comb drives can be utilized to generate large out-of-plane or torsional motions [11]. Generally speaking, in a typical in-plane interdigitated comb drive, the capacitance is linear with displacement, resulting in an electrostatic driving force, which is independent of the position of the movable fingers except at the ends of the range of travel [12]. However, for some special applications, a comb drive with variable-gap profiles can be designed that will deliver desired driving force profiles by solving an appropriate inverse problem [13]. Because the driving force will seriously affect the performance and reliability

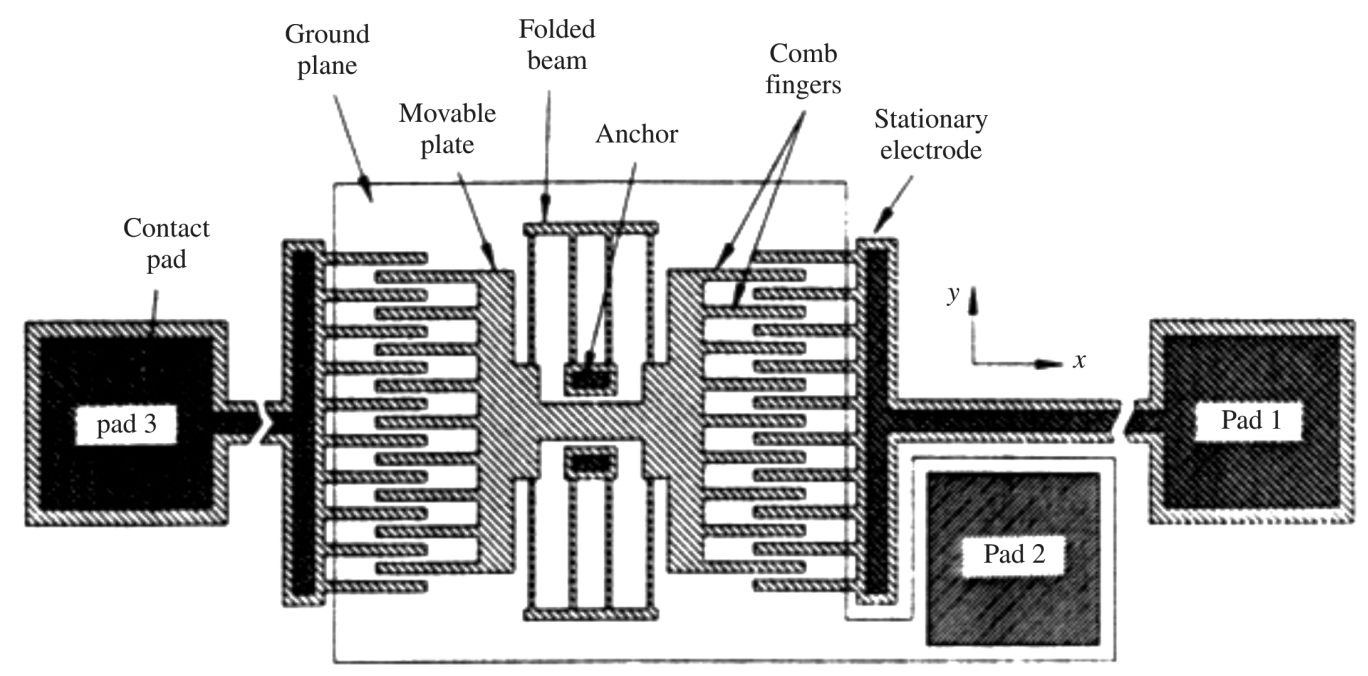

Fig. 3 Layout of a linear lateral resonator driven and sensed with interdigitated capacitors (electrostatic comb drive) [8] 
of MEMS devices, how to obtain the actual electrostatic force during the travelled process plays a very important role. As a result, studies of the effects of the gap, width ratio between fixed and movable fingers, and distance travelled by movable finger considering the fringing field around the edges are very important for engineers and scientists.

Basically, electrical engineers are familiar with electrostatic problems, and various numerical methods have been regularly used in MEMS and electromagnetics [14]. Among different numerical approaches, the finite element method (FEM), which is based on the representation and approximate solution of boundary value problems of engineering mathematics in terms of partial differential equations $[\mathbf{1 5}, \mathbf{1 6}]$, and the boundary element method (BEM) based on integral equations [17] have moved from being research tools for scientists to becoming powerful design tools for engineers. One of the main advantages of the BEM, when compared with the FEM, is that discretizations are restricted only to the boundaries, making data generation much easier. The BEM is also ideally suited to the analysis of external problems where domains extend to infinity, since discretizations are confined to the internal boundaries with no need to truncate the domain at a finite distance and to impose artificial boundary conditions. It is appropriate for problems involving some form of discontinuity or singularity as well, due to the use of singular fundamental solutions as test functions. It is also interesting to point out that the unknowns in the BEM are a mixture of the potential and its normal derivative, rather than the potential only as in the FEM. This is a consequence of the fact that the BEM is a 'mixed' formulation and constitutes an important advantage over the FEM. In particular, for various gaps between the fixed and movable fingers of the comb drive of the MEMS, many laborious studies of finite element modelling compared with those of boundary element modelling are needed because the BEM can provide a complete solution in terms of boundary values, with substantial saving in modelling effort [18]. Because the dual boundary element method (DBEM) is a very efficient new method for solving the electrostatic problems of electromagnetics [19] and MEMS [20], the DBEM was used to simulate the fringing field around the edges of the fixed and movable fingers of the MEMS comb drive for various design cases in this article.

The paper is organized as follows. Section 2 involves a review of the approximate method for calculating the driving force. In section 3, the procedure for the dual integral equation for MEMS electrostatic problems is concisely introduced. Before the DBEM was used to solve and simulate real MEMS devices and structures, a benchmark test was performed against the analytical solution to compare its computational accuracy in section 4. Numerical results are provided and compared in section 5 to study the effects of various gaps, finger

C01304 (C) IMechE 2004 width ratios and travelled distances for driving force acting on the movable finger. Some remarks based on the reported results are discussed in section 6. Finally, there is a concise conclusion in section 7 .

\section{REVIEW OF APPROXIMATE METHOD FOR CALCULATING THE DRIVING FORCE}

It is well known that the driving force due to the electrostatic field is very important for the MEMS performance [13]. Traditionally the fringing field around the edge of comb drive fingers was not considered, and an approximate formula was widely adopted for calculating the driving force acting on the movable finger to simplify the analytical task. However, it does not mean that the fringing effect is not useful and could be neglected arbitrarily. Instead, this effect usually plays a very important role for some critical design. From reference [21], the driving force acting on the movable finger can be approximately calculated using a capacitance model. This approximate formula, which is widely used in practice, is

$$
F=\frac{\varepsilon h V^{2}}{g}
$$

where $F$ is the driving force acting on the movable finger, $\varepsilon$ is the permittivity of the medium, $h$ is the height of the movable finger (in a direction normal to Fig. 4), $V$ is the bias voltage and $g$ is the gap between fixed and movable fingers. The direction of the force is parallel to the direction of the fingers and causes the opposing halves to attract each other. In this idealized model, the force does not depend on the deflection. Basically, equation (1) is accurate for overlap travelled distances greater than $2 g$ and less than $3 g$ from the point where the comb fingers reach the back end of the actuator [21].

Although the approximate formula given in equation (1) can be used for calculating the driving force of the comb drive of the MEMS, there are still some fringing fields around the edge of the movable finger for various gaps and also the travelled distances are not taken into account. In order to obtain more reasonable computational results for the electric field, the DBEM is

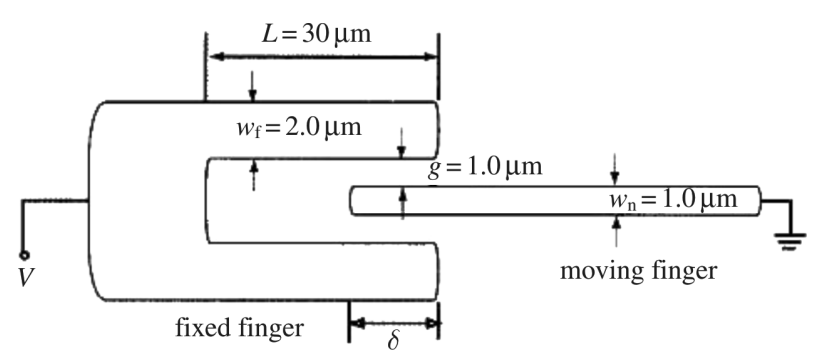

Fig. 4 A prototype comb drive with one set of straight fingers 
employed to analyse electrostatic problems for the comb drive of the MEMS considering the fringing field around the edges in the article. After using the DBEM to calculate accurately the electrostatic response of the comb fingers biased with a d.c. voltage, the induced driving force acting on the movable comb finger at different gaps, finger width ratios and travelled distances can be obtained. Then this driving force can be plotted against distance travelled by the movable finger and gaps between fixed and movable fingers.

\section{DUAL INTEGRATION EQUATION FOR EXTERIOR MEMS ELECTROSTATIC PROBLEMS}

Generally the electrostatic problem consists of finding the unknown potential (voltage) function $\boldsymbol{V}$ in the partial differential equation. In addition to the fact that $\boldsymbol{V}$ satisfies $\nabla^{2} \boldsymbol{V}=\mathbf{0}$ within a prescribed solution region $\Omega$, the potential function $\boldsymbol{V}$ must satisfy certain conditions on $B$ which is the boundary of $\Omega$. Usually these boundary conditions are the Dirichlet $[\boldsymbol{V}(\boldsymbol{x})=f(\boldsymbol{x})]$ and Neumann $\left[\partial \boldsymbol{V}(\boldsymbol{x}) / \partial \boldsymbol{n}_{\boldsymbol{x}}=g(\boldsymbol{x})\right]$ types, where $f(\boldsymbol{x})$ and $g(\boldsymbol{x})$ denote known boundary data, and $\boldsymbol{n}_{\boldsymbol{x}}$ is the unit outer normal vector at the point $\boldsymbol{x}$ on the boundary $\boldsymbol{B}$. Therefore, the governing equation of electrostatic problems could be written in the form

$$
\nabla^{2} \boldsymbol{V}(\boldsymbol{x})=\mathbf{0}, \quad \boldsymbol{x} \text { in } \Omega
$$

Based on the dual boundary integral equation formulation for the electrostatic problem $[\mathbf{1 9}, \mathbf{2 0}]$,

$$
\begin{aligned}
\alpha \boldsymbol{V}(\boldsymbol{x})= & \mathrm{CPV} \int_{\boldsymbol{B}} \boldsymbol{T}(\boldsymbol{s}, \boldsymbol{x}) \boldsymbol{V}(\boldsymbol{s}) \mathrm{d} \boldsymbol{B}(\boldsymbol{s}) \\
& -\mathrm{RPV} \int_{\boldsymbol{B}} U(\boldsymbol{s}, \boldsymbol{x}) \frac{\partial \boldsymbol{V}(\boldsymbol{s})}{\partial \boldsymbol{n}_{\boldsymbol{s}}} \mathrm{d} \boldsymbol{B}(\boldsymbol{s}) \\
\alpha \frac{\partial \boldsymbol{V}(\boldsymbol{x})}{\partial \boldsymbol{n}_{\boldsymbol{x}}}= & \operatorname{HPV} \int_{\boldsymbol{B}} M(\boldsymbol{s}, \boldsymbol{x}) \boldsymbol{V}(\boldsymbol{s}) \mathrm{d} \boldsymbol{B}(\boldsymbol{s}) \\
& -\mathrm{CPV} \int_{\boldsymbol{B}} \boldsymbol{L}(\boldsymbol{s}, \boldsymbol{x}) \frac{\partial \boldsymbol{V}(\boldsymbol{s})}{\partial \boldsymbol{n}_{\boldsymbol{s}}} \mathrm{d} \boldsymbol{B}(\boldsymbol{s})
\end{aligned}
$$

where the kernel functions $U(\boldsymbol{s}, \boldsymbol{x})=\ln (r), \quad \boldsymbol{T}(\boldsymbol{s}, \boldsymbol{x})=$ $\partial U(\boldsymbol{s}, \boldsymbol{x}) / \partial \boldsymbol{n}_{\boldsymbol{s}}, \quad \boldsymbol{L}(\boldsymbol{s}, \boldsymbol{x})=\partial U(\boldsymbol{s}, \boldsymbol{x}) / \partial \boldsymbol{n}_{\boldsymbol{x}}, \quad M(\boldsymbol{s}, \boldsymbol{x})=$ $\partial^{2} U(\boldsymbol{s}, \boldsymbol{x}) / \partial \boldsymbol{n}_{\boldsymbol{x}} \partial \boldsymbol{n}_{\boldsymbol{s}}, \quad r=|\boldsymbol{s}-\boldsymbol{x}|, \boldsymbol{s}$ and $\boldsymbol{x}$ being position vectors of the points $s$ and $x$ respectively, and $\boldsymbol{n}_{\boldsymbol{s}}$ is the unit outer normal vector at point $\boldsymbol{s}$ on the boundary. In addition, RPV is the Riemann principal value, CPV is the Cauchy principal value, HPV is the Hadamard principal value and $\alpha$ depends on the collocation point $(\alpha=2 \pi$ for an interior point, $\alpha=\pi$ for a smooth boundary and $\alpha=0$ for an exterior point). The commutativity property of the trace operator and the normal derivative operator provides alternative ways to calculate the Hadamard principal value analytically. Equation (3) is called the singular boundary integral equation and equation (4) is called the hypersingular boundary integral equation. Since the hypersingular boundary integral equation plays an important role in degenerate problems, many researchers have paid much attention to this. After discretizing the boundary into $2 N$ boundary elements, equations (3) and (4) are reduced to

$$
\begin{aligned}
& {[U]_{2 N \times 2 N}\{t\}_{2 N \times 1}=[T]_{2 N \times 2 N}\{u\}_{2 N \times 1}} \\
& {[L]_{2 N \times 2 N}\{t\}_{2 N \times 1}=[M]_{2 N \times 2 N}\{u\}_{2 N \times 1}}
\end{aligned}
$$

where $[U],[T],[L]$ and $[M]$ are the four influence matrices, and $\{u\}$ and $\{t\}$ are the boundary data for the primary and the secondary boundary variables respectively.

\section{AN EXAMPLE OF A BENCHMARK TEST FOR DBEM}

Before the DBEM was used to solve and simulate real MEMS devices and structures, the following benchmark test was performed for comparison with the analytical solution to verify its computational accuracy.

\subsection{Benchmark test}

Two grounded semi-infinite parallel-plate electrodes are separated by a distance $b$. A third electrode perpendicular to and insulated from both is maintained at a constant potential $V_{0}$ (Fig. 5). Determine the electric potential distribution in the region enclosed by the electrodes.

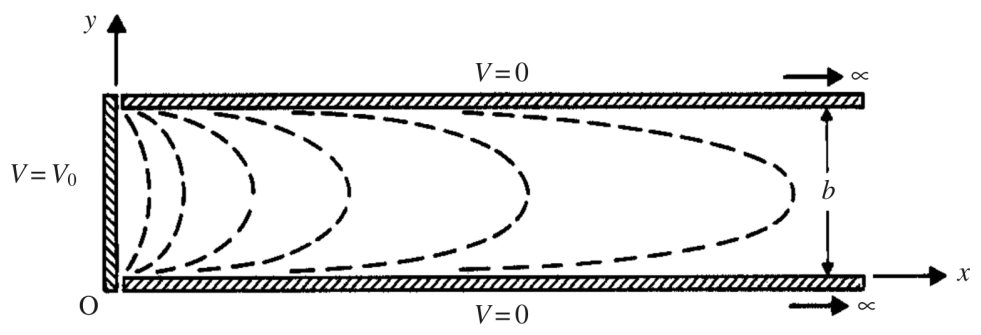

Fig. 5 Cross-sectional figure for the benchmark test. The plane electrodes are infinite in the $z$ direction 


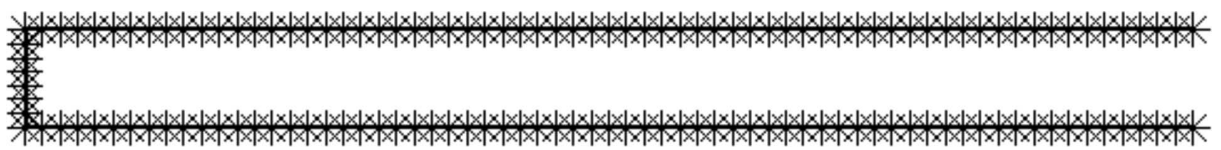

Fig. 6 The related DBEM mesh discretization of the benchmark test (146 elements and 146 nodes)

\subsection{Analytical solution}

Using the method of separation of variables, the desired electric potential distribution $V(x, y)$ could be obtained [14]:

$$
V(x, y)=\Sigma C_{n} \exp \left(\frac{-n \pi x}{b}\right) \sin \left(\frac{n \pi y}{b}\right)
$$

where $C_{n}=4 V_{0} / n \pi$ if $n$ is odd and $C_{n}=0$ if $n$ is even; $n=1,3,5, \ldots, \infty$. Equation (7) is a rather complicated expression to plot but, since the amplitude of the sine terms in the series decreases very rapidly as $n$ increases, only the first few terms are needed to obtain a good approximation. Several equipotential lines are sketched in Fig. 5.

\subsection{DBEM analysis}

For convenience, the value of $b$ is assumed to be 3.333 , and four points will be analysed using rough mesh discretization [146 elements and 146 nodes (Fig. 6)] of the DBEM, and compared with analytical data computed from equation (7). The results of electric potential under DBEM and analytical methods are listed in Table 1. Because the errors between analytical method and DBEM are lower than 1 per cent, the simulation data for the DBEM presented in this article are valid and convincing for electrical engineers.

\section{DBEM SIMULATION OF VARIATIONS IN GAP AND FINGER WIDTH RATIO AND TRAVELLED DISTANCE FOR THE DRIVING FORCE OF THE COMB DRIVE OF AN MEMS}

In order to investigate the effects of variations in the gap and finger width ratio (between movable and fixed fingers) and the distance travelled by the movable finger

Table 1 The results of electric potential of benchmark test under DBEM and analytical methods

\begin{tabular}{llll}
\hline & \multicolumn{2}{c}{$V(x, y)$} & \\
\cline { 2 - 3 } $\begin{array}{l}\text { Locations } \\
(x, y)\end{array}$ & $\begin{array}{l}\text { From } \\
\text { DBEM }\end{array}$ & $\begin{array}{l}\text { From the analytical } \\
\text { method }\end{array}$ & $\begin{array}{l}\text { Error } \\
(\%)\end{array}$ \\
\hline$(0.667,2.778)$ & $0.40500 V_{0}$ & $0.407890 V_{0}$ & -0.71 \\
$(3.333,0.556)$ & $0.027363 V_{0}$ & $0.027536 V_{0}$ & -0.63 \\
$(6.667,1.111)$ & $0.002043 V_{0}$ & $0.002058 V_{0}$ & -0.73 \\
$(9.999,1.667)$ & $0.000102 V_{0}$ & $0.000103 V_{0}$ & -0.97 \\
\hline
\end{tabular}

for the driving force acting on the movable finger of the comb drive of an MEMS, the following cases were studied and compared with the results obtained from the approximate method shown in equation (1).

\subsection{Case 1}

A prototype comb drive with one set of straight fingers (with differential d.c. bias $V$ ) shown in Fig. 4 was considered. In this case, the widths of movable and fixed fingers are $1.0 \mu \mathrm{m}\left(w_{n}\right)$ and $2.0 \mu \mathrm{m}\left(w_{f}\right)$, the gap $g$ between the fixed and movable fingers is $1.0 \mu \mathrm{m}$, and the height of the movable finger is $h(h / g \geqslant 10)$. The driving force under different travelled distance $\delta$ (from -5.0 to $29.5 \mu \mathrm{m})$ and the difference between the DBEM and the approximate method needed to be calculated and investigated.

From the drawing of a comb drive in which the electric field lines are sketched (Fig. 7), it can be seen that there will be an obvious fringing field around the edge of the movable finger, and the physical behaviour (e.g. electric potential and electric field intensity) of this area is very complicated. Since it is not easy to obtain the analytical solutions, and some simplified numerical models for electrostatic comb drives from references [11] and [12] cannot accurately simulate the fringing field, a large FEM model was commonly used in industry for the exterior electrostatic problems of the MEMSs. As is known, MEMS structures usually have a very high aspect ratio; for example, when the ratio of the height to gap between fingers in the comb drives is of the order of $10(h / g \geqslant 10)$, hence, the comb drive can be approximated to be infinitely long in the $z$ direction (in a

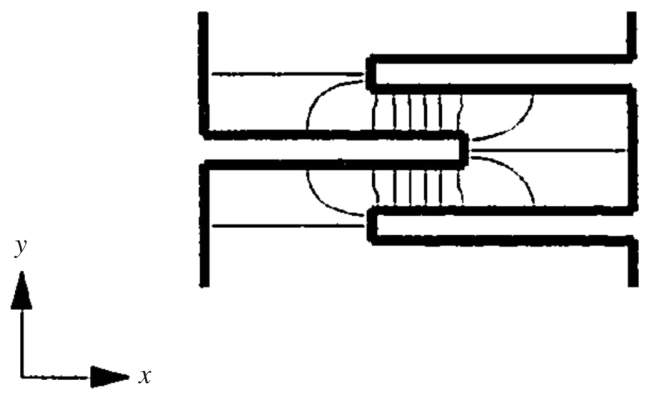

Fig. 7 Drawing of a comb drive with electric field lines sketched. The net force acting on the left finger is in the $+\boldsymbol{x}$ direction. A voltage difference exists between the single finger on the left and the pair of fingers on the right 


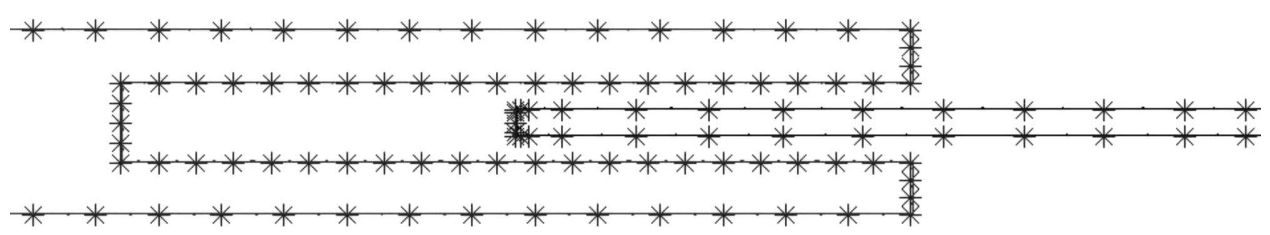

Fig. 8 The related DBEM coarse mesh discretization

direction normal to Fig. 4). Based on this assumption, a two-dimensional model of this problem is proposed here. For problems with variable $g$, finger width ratio $R\left(=w_{f} / w_{n}\right)$ and $\delta$ of the comb drive of the MEMS, since the boundary-type DBEM has an advantage over the domain-type FEM because the DBEM can provide a complete solution in terms of boundary values, with substantial saving in modelling effort [19], the DBEM was used to simulate the fringing field around the edges of the fixed and movable fingers of the comb drive of the MEMS in this case, and the electric field can be accurately calculated using coarse DBEM mesh discretization [132 elements and 132 nodes (Fig. 8)].

Because the charge distribution $\rho_{\mathrm{s}}$ on the conductor surfaces can be determined from $\rho_{\mathrm{s}}=\varepsilon E_{\mathrm{n}}$ (i.e. the normal component of the electric field, $E_{\mathrm{n}}$, at a conductor boundary is equal to the surface charge density $\rho_{\mathrm{s}}$ on the conductor divided by the permittivity $\varepsilon$ ), if $\varepsilon$ is a constant, the relationship between the normal force density $f_{\mathrm{n}}$ acting on the surface of a conductor and the charge density $\rho_{\mathrm{s}}$ of that conductor is [14]

$$
f_{\mathrm{n}}=\frac{-0.5 \rho_{\mathrm{s}}^{2}}{\varepsilon}
$$

Thus, the electrostatic force density $F_{\mathrm{n}}$ acting on the movable finger along the boundary

$$
F_{\mathrm{n}}=\int_{\boldsymbol{B}} \boldsymbol{f} \mathrm{d} \boldsymbol{B}
$$

can be calculated if $\rho_{\mathrm{s}}$ (or $E_{\mathrm{n}}$ ) is known. Therefore, the driving force density $F$ acting on the movable finger for various values of $\delta$ can be calculated, and the $F$ acting on the movable finger is shown in Fig. 9. The difference $F_{\text {DBEM }} / F_{\text {Approx }}-1$ and absolute difference values between the DBEM and approximate method for various $\delta$ are also shown in Figs 10 and 11 respectively. The results show that the absolute difference values are about 10 per cent while the value of $\delta$ is greater than $2 g$ and less than $3 g$ from the point where the comb fingers reach the back end of the actuator, and the error of the approximate method becomes much larger because of the complex fringing effect at the beginning and ends of the range of travel. Even if the driving force acting on the movable finger is almost independent of the position of the movable finger except at the beginning and ends of the range of travel, the approximate method cannot be efficiently used due to inaccuracy.

\subsection{Case 2}

The effect of the gap between fixed and movable fingers will be investigated in this case, because it may play an important role for the distribution of driving force. For simplification, $\delta$ is confined to $15 \mu \mathrm{m}$, but the values of $g$ are variable from 0.8 to $4 \mu \mathrm{m}$. If a movable comb finger with a differential d.c. bias $V$ is also applied to the two adjacent electrodes as shown in Fig. 4, it is required to determine the distribution of $F$ acting on the movable finger and the approximate error.

Using the DBEM, the distribution of the normal electric field intensity $E_{\mathrm{n}}$ on the movable finger for various values of $g$ can be precisely obtained, and the

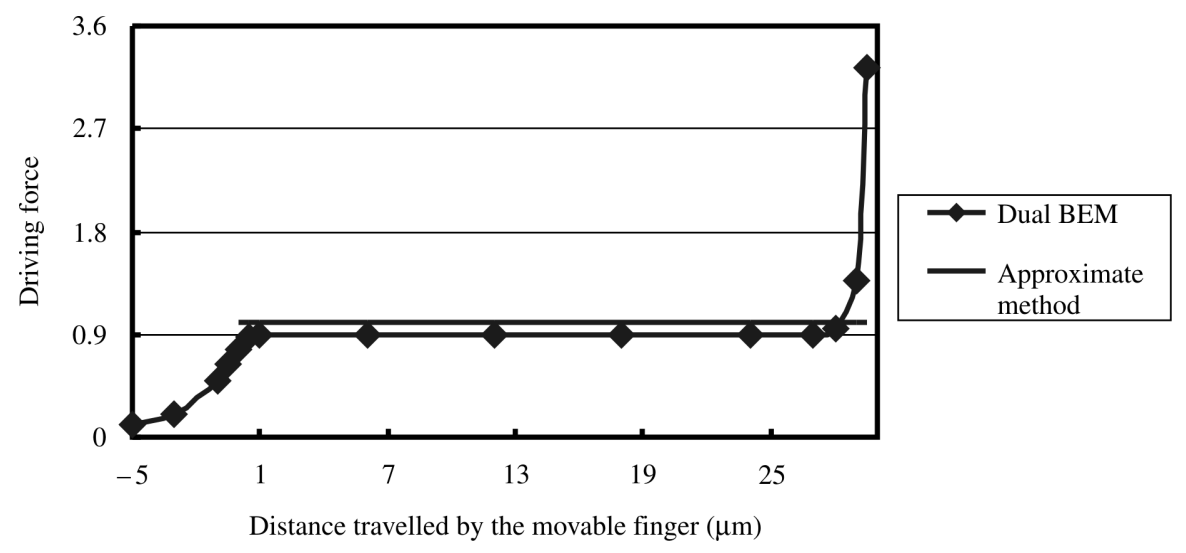

Fig. 9 The distribution of driving force acting on the movable finger for various distances travelled by the movable finger (units, $s h V^{2}$ ) 


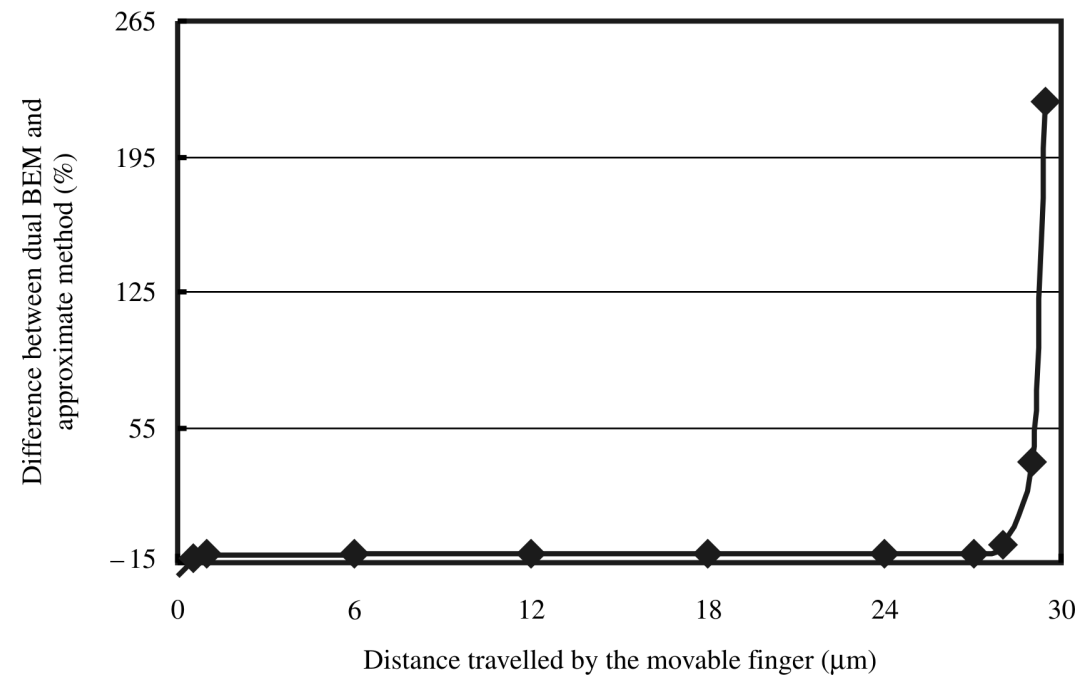

Fig. 10 The difference between the DBEM and the approximate method for various distances travelled by the movable finger

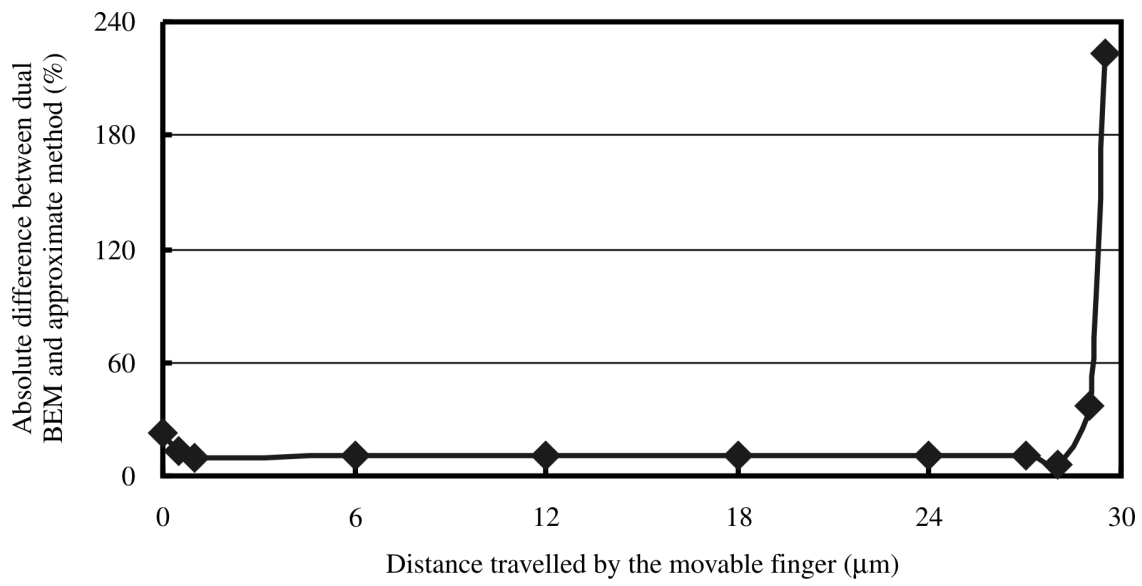

Fig. 11 The absolute difference value between the DBEM and the approximate method for various distances travelled by the movable finger

charge distribution on the conductor surfaces can also be determined from $\rho_{\mathrm{s}}=\varepsilon E_{\mathrm{n}}$. Using equations (8) and (9), the distribution of $F$ for different $g$ is computed and shown in Fig. 12. The difference and absolute difference values between the DBEM and the approximate method for different $g$ are shown in Figs 13 and 14 respectively. The results show that, the smaller the gap, the larger is the driving force and the error of approximate method also becomes more and more predominant as the gap between the movable and fixed fingers decreases. Therefore, care should be taken by engineers and scientists using the approximate method for various $g$.

\subsection{Case 3}

Besides the effect of $g$, the width ratio between fixed and movable fingers for various $\delta$ also needed to be investigated. In this case, the values of $R\left(=w_{\mathrm{f}} / w_{\mathrm{n}}\right)$ are variable from 3 to $1 / 3$, but $g$ is still confined to $1.0 \mu \mathrm{m}$. If a movable comb finger with a differential d.c. bias $V$ is applied to the two adjacent electrodes shown in Fig. 4, it is necessary to determine $F$ and the error of the approximate method as in the previous case.

Using the DBEM, the distribution of $E_{\mathrm{n}}$ on the movable finger for various values of $R$ can also be reasonably calculated, and $\rho_{\mathrm{s}}$ on the conductor surfaces can be determined as well. By using equations (8) and (9), the distribution of $F$ for different $R$ and various $\delta$ is computed and given in Fig. 15. The difference and absolute difference values between the DBEM and the approximate method for different $R$ are also obtained and shown in Figs 16 and 17 respectively. It can be seen from these figures that the difference between the DBEM and the approximate method effect due to finger width ratio is very small. 


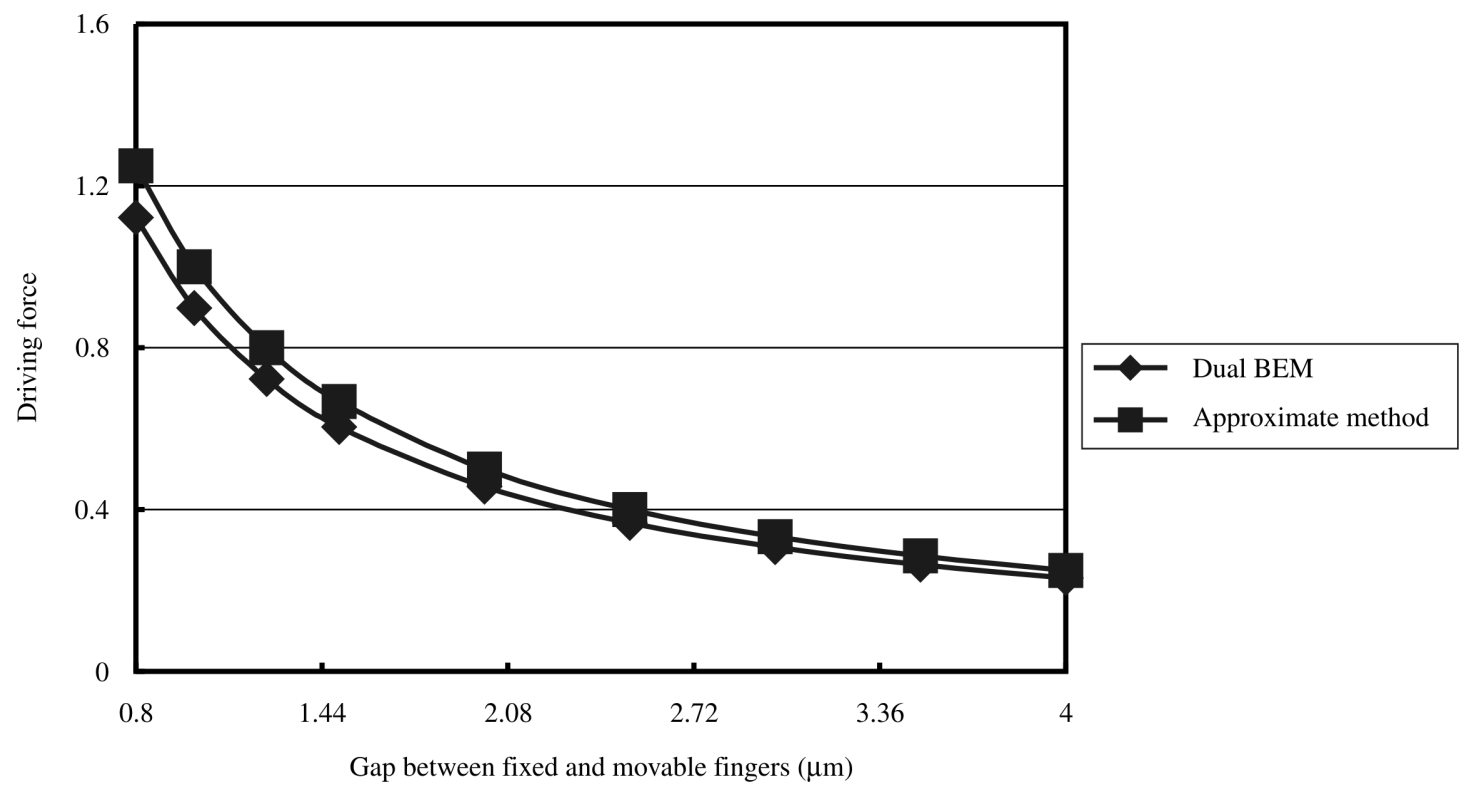

Fig. 12 The distribution of driving force acting on the movable finger for various gaps between the fixed and movable fingers (units, $\varepsilon h V^{2}$ )

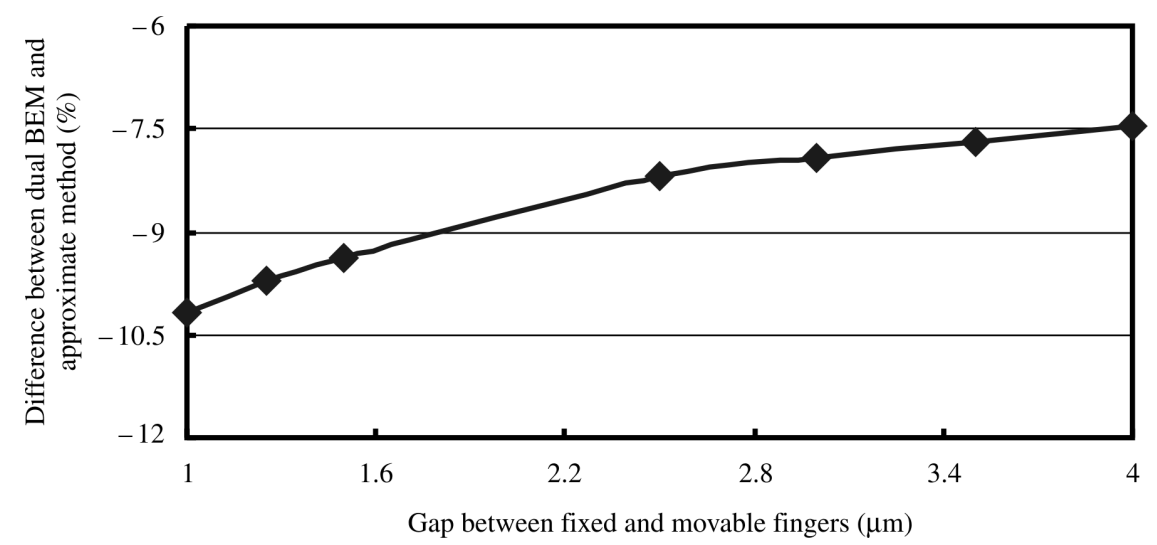

Fig. 13 The difference between the driving forces for the DBEM and the approximate method for various gaps between the fixed and movable fingers

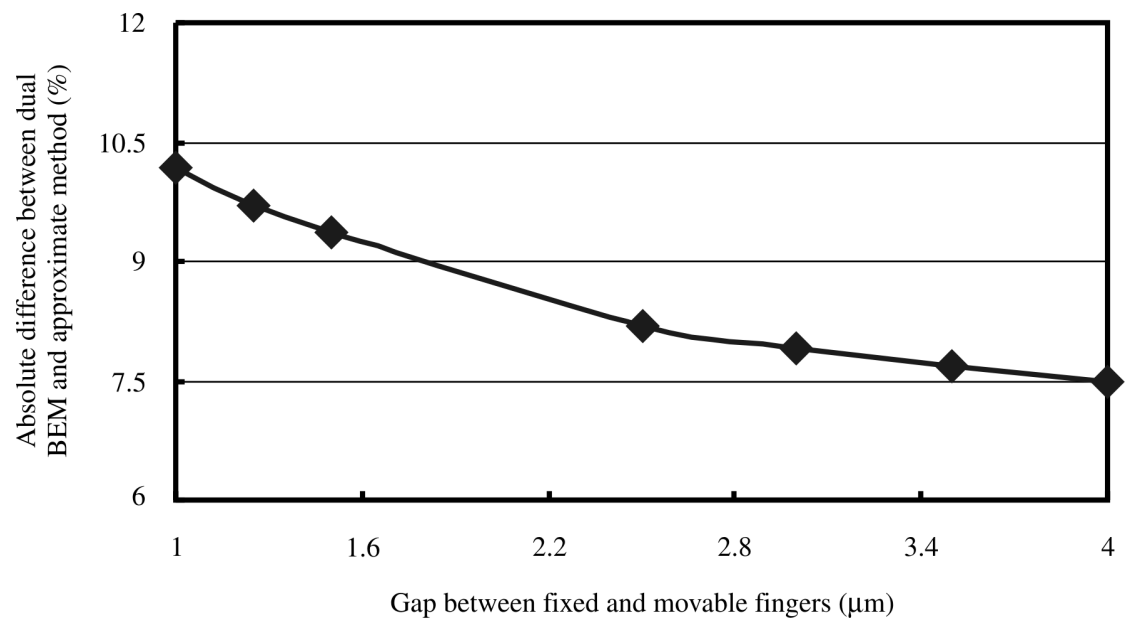

Fig. 14 The absolute difference value between the driving forces for the DBEM and the approximate method for various gaps between the fixed and movable fingers 


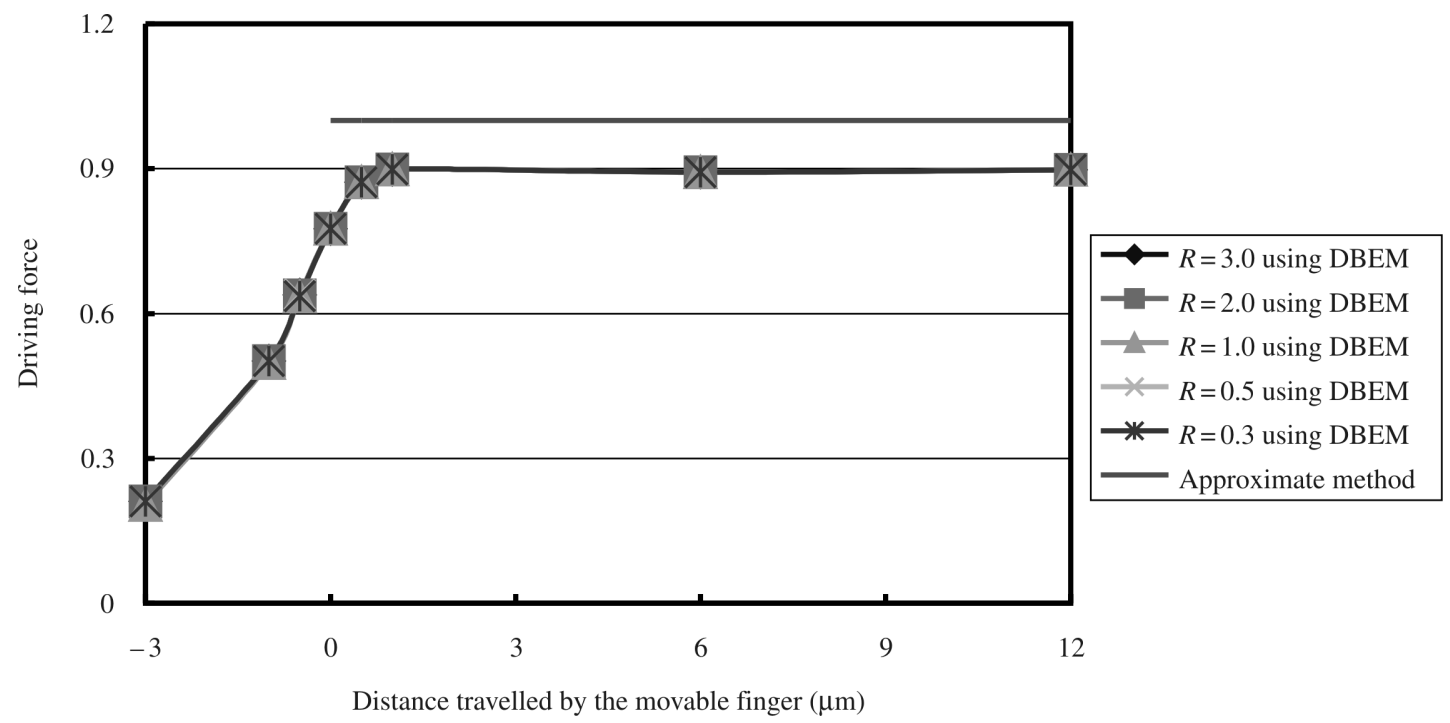

Fig. 15 The distribution of the driving force for various travelled distances, gaps and finger width ratios (units, $\varepsilon h V^{2}$ )

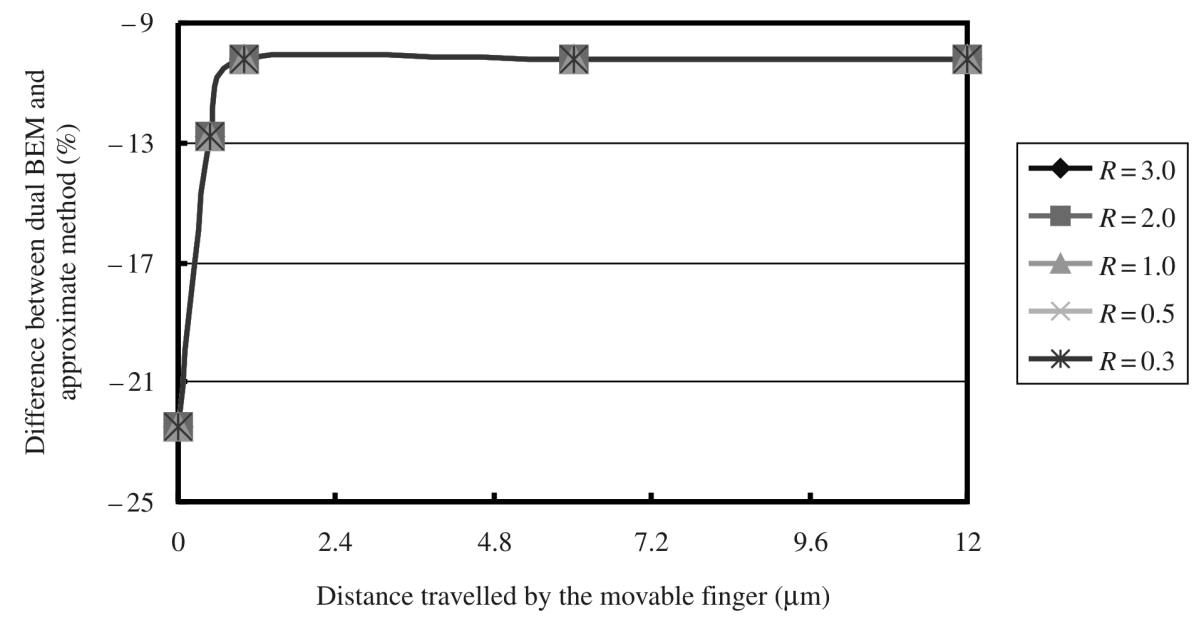

Fig. 16 The difference between the driving forces for the DBEM and the approximate method for various finger width ratios and travelled distances

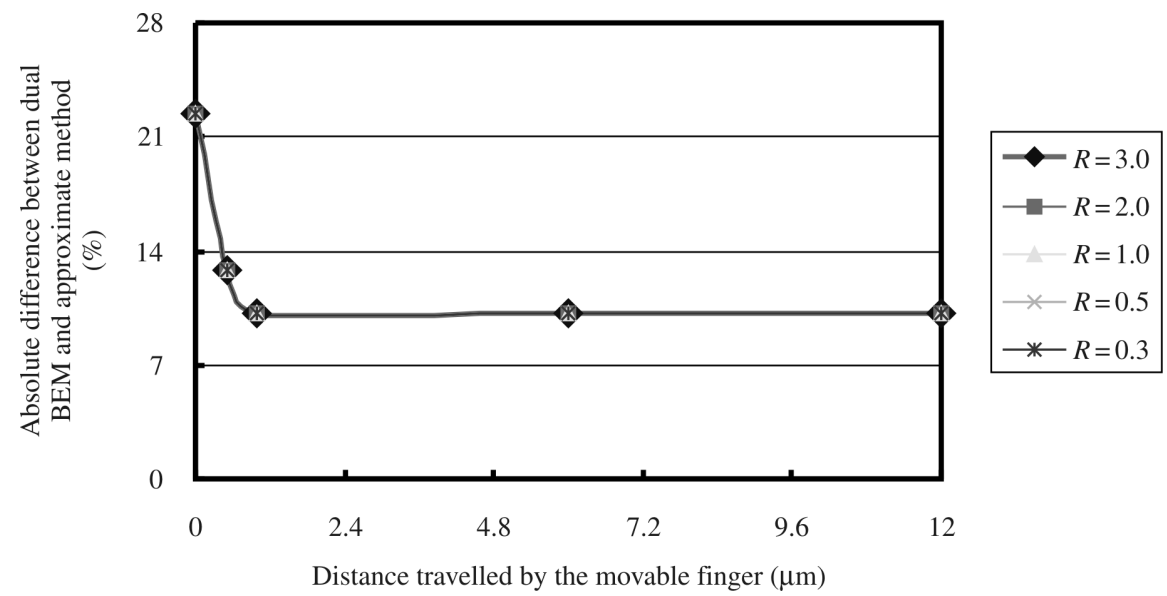

Fig. 17 The absolute difference value between the driving forces for the DBEM and the approximate method for various finger width ratios and travelled distances 


\section{DISCUSSION}

1. Because the simulation of the driving force acting on the movable is indispensable for the comb drive, the way in which the electrostatic field can be accurately obtained is very important for the design of MEMS devices. Although an approximate method has been widely used for convenience, it cannot always work well for many cases because the fringing fields are not taken into account, which may result from the gap $g$ between fixed and movable fingers and the width ratio $R$ of the fixed finger to the movable finger. After using the DBEM to calculate accurately the electrostatic response of the comb fingers biased with a d.c. voltage, the driving force $F$ acting on the movable finger at different travelled distances $\delta$ and $g$ can be obtained and investigated.

2. The results show that the absolute difference values are about 10 per cent while the value of $\delta$ is greater than $2 g$ and less than $3 g$ from the point where the comb fingers reach the back end of the actuator, and the error of the approximate method becomes much larger because of the complex fringing effect at the beginning and ends of the range of travel. Even if the driving force acting on the movable finger is almost independent of the position of the movable finger except at the beginning and ends of the range of travel, the approximate method cannot be efficiently used due to inaccuracy.

3. It is found that, the smaller the gap, the larger is the driving force and the error of approximate method also becomes more and more predominant as the gap between the movable and fixed fingers decreases. Therefore, care should be taken by engineers and scientists using the approximate method for various $g$. In addition, the difference between the DBEM and the approximate method effect due to the finger width ratio is very small.

4. Although using the FEM is widespread for MEMS devices nowadays, it is still very difficult to establish the boundary conditions and to generate all the proper FEM because the design parameters of the comb drive of the MEMS (the gaps between fingers and the finger width ratios) always change many times before the final layout in the variable design stage. Therefore, the DBEM is strongly recommended for studying the electrostatic field of the comb drive of an MEMS because the DBEM discretizations are restricted only to the boundaries, which makes data generation much easier than with the FEM.

5. From reference 10, the quality factor of the comb drive, $Q$, is proportional to the resonant frequency of the comb drive, and the resonant frequency is obviously dependent on the driving force because the stiffness and resonant frequency can be tuned by applying a voltage to the actuator. Therefore, more accurate numerical data on the resonant frequency and quality factor than found by the conventional approximate method can be obtained in the design stage if the work presented in this article is implemented.

\section{CONCLUSIONS}

The fringing field around the edge of the comb drive of an MEMS is important for calculating the driving force acting on the movable finger. The dual integral formulation of electrostatic comb-drive problems considering the fringing field around the edges has been presented in this article because the approximate method cannot work well for many cases. For electrical engineering practices, much time is needed for variable design if using the domain-type FEM; therefore the present boundary-type DBEM has great potential for industrial applications, especially in the initial design stage. Therefore, the follow-up control method of driving force for the comb drive of an MEMS can be implemented more precisely.

\section{REFERENCES}

1 Varadan, V. K., Vinoy, K. J. and Jose, K. A. $R F$ MEMS and Their Applications, 2003 (John Wiley, New York).

2 Tilmans, H. A. C., Raedt, W. D. and Beyne, E. MEMS for wireless communications: from RF-MEMS components to RF-MEMS-SiP. J. Micromech. Microengng, 2003, 13, S139-S163.

3 Nguyen, C. T. C., Katechi, L. P. B. and Rebeiz, G. M. Micromachined devices for wireless communications. Proc. IEEE, 1998, 86, 1756-1768.

4 Rebeiz, G. M. and Muldavin, J. B. RF MEMS switches and switch circuits. IEEE Microwave Mag., 2001, 2, 59-71.

5 Yao, J. J. RF MEMS from a device perspective. J. Micromech. Microengng, 2000, 10, R9-R38.

6 Yao, J. J., Park, S. T. and DeNatale, J. High tuning-ratio MEMS-based tunable capacitors for RF communications applications. In Proceedings of the Solid-State Sensor and Actuator Workshop, Hilton Head, South Carolina, 8-11 June 1998, pp. 124-127.

7 van Spengen, W. M., Puers, R., Mertens, R. and De Wolf, I. A comprehensive model to predict the charging and reliability of capacitive RF MEMS switches. J. Micromech. Microengng, 2004, 14, 514-521.

8 Tang, W. C., Nguyen, T. C. H., Judy, M. W. and Howe, R. T. Lateral driven polysilicon resonant microstructures. Sensors and Actuators, 1989, 20, 25-32.

9 Tang, W. C., Nguyen, T. C. H., Judy, M. W. and Howe, R. T. Electrostatic-comb drive of lateral polysilicon resonators. Sensors and Actuators A, 1990, 21-23, 328-331.

10 Tang, W. C., Lim, M. G. and Howe, R. T. Electrostatic comb drive levitation and control method. IEEE J. Microelectromech. Systems, 1992, 1(4), 170-178. 
11 Yeh, J. L. A., Hui, C. Y. and Tien, N. C. Electrostatic model for an asymmetric combdrive. IEEE J. Microelectromech. Systems, 2000, 9(1), 126-135.

12 Johnson, W. A. and Warne, L. K. Electrophysics of micromechanical comb actuators. IEEE J. Microelectromech. Systems, 1995, 4(1), 49-59.

13 Ye, W., Mukherjee, S. and MacDonald, N. C. Optimal shape design of an electrostatic comb drive in microelectromechanical systems. IEEE J. Microelectromech. Systems, 1998, 7(1), 16-26.

14 Cheng, D. K. Field and Wave Electromagnetics, 1989 (Addison-Wesley, Reading, Massachusetts).

15 Beerschwinger, U., Miline, N. G., Yang, S. J., Reuben, R. L., Sangster, A. J. and Ziad, H. Coupled electrostatic and mechanical FEA of a micromotor. IEEE J. Microelectromech. Systems, 1994, 3(4), 162-171.

16 Silvester, P. P. and Ferrari, F. L. Finite Elements For Electrical Engineers, 1983 (Cambridge University Press, Cambridge).
17 Harrington, R. F. and Sarkar, T. K. Boundary elements and the method of moments. In Boundary Elements (Eds C. A. Brebbia, T. Futagami and M. Tanaka), 1983, pp. 31-40 (CML, Southampton).

18 Senturia, S. D., Aluru, N. and White, J. Simulating the behavior of MEMS devices: computational methods and needs. IEEE Comput. Sci. Engng Mag., January-March 1997, 30-43.

19 Chyuan, S. W., Liao, Y. S. and Chen, J. T. An efficient method for solving electrostatic problems. IEEE Comput. Sci. Engng Mag., May-June 2003, 52-58.

20 Liao, Y. S., Chyuan, S. W. and Chen, J. T. An alternatively efficient method for simulating the electrostatic field and levitating force of MEMS combdrive. J. Micromech. Microengng, 2004, 14, 1258-1269.

21 Adams, S. Design of electrostatic actuators to tune the effective stiffness of microelectromechanical systems. PhD thesis, Cornell University, Ithaca, New York, 1996. 